Research Article

\title{
Chattering-Free Reference Sliding Variable-Based SMC
}

\author{
Katarzyna Adamiak (iD \\ Institute of Automatic Control, Łódź University of Technology, Łódź 90-924, Poland \\ Correspondence should be addressed to Katarzyna Adamiak; katarzyna.adamiak@dokt.p.lodz.pl
}

Received 22 June 2020; Revised 1 August 2020; Accepted 10 August 2020; Published 7 September 2020

Academic Editor: Denizar Cruz Martins

Copyright (C) 2020 Katarzyna Adamiak. This is an open access article distributed under the Creative Commons Attribution License, which permits unrestricted use, distribution, and reproduction in any medium, provided the original work is properly cited.

\begin{abstract}
The paper addresses the problem of chattering elimination in sliding mode control for sampled data dynamical systems and proposes an innovative control scheme. The key to the proposed control method is utilizing a pregenerated nonswitching type reference sliding variable profile to control the disturbed plant. As sampled data systems contain sample-and-hold devices in their input and output channels, we carry out a discrete time analysis. We consider the discretization effects on the controller in two aspects: the pace of convergence of the system and the ultimate band width. Consequently, in the reference sliding variable generator, we analyse two reaching laws, differently adapting to changes of the controller's frequency. We prove that this approach not only minimizes the chattering phenomenon but also provides a reduction of the quasi-sliding mode band width, which in general case remains of $O(T)$ order. Furthermore, the proposed control method incorporates a disturbance compensation algorithm, which results in the ultimate band width of $O\left(T^{2}\right)$ order. Finally, we also show that a certain selection of the sliding plane guarantees limitation of all the state variables' errors to $O\left(T^{2}\right)$ order as well. Therefore, the proposed control algorithm significantly improves the system's robustness.
\end{abstract}

\section{Introduction}

Nowadays, it is hard to imagine any control implementation without microprocessors, digital sensors, and actuators. All such devices operate in sample-and-hold manner. Namely, they measure or calculate the signal's value in the current moment $k$ and hold this value until the next measurement, in moment $k+1$, is done. Therefore, they enforce discretization of all input and output signals. Consequently, researchers over the world are forced to study the discretization's impact on the system's dynamics and design discrete time controllers. One of the branches of discrete time control engineering has become the quasi-sliding mode control.

Although continuous time sliding mode is a comparably new control idea, it has been thoroughly studied since the 1970s [1-3]. It assumes continuous switching of the structure of the controller depending on the position of the representative point of the system relative to the chosen sliding plane so that the system's states are always driven onto the plane. Once the representative point of the system becomes confined to the sliding plane, it slides along it to the equilibrium point. Therefore, the stability of the system depends on the choice of the sliding plane only. The idea of sliding mode control has quickly gained popularity [4-7]; thanks to continuous updates of the control signal, it ensures insensitivity to matched external disturbance and model uncertainties [8].

Unfortunately, the digital implementation of sliding mode causes new issues to arise. As the frequency of the controller becomes limited, it is unable to maintain the system's motion on the sliding plane. Consequently, between consecutive control updates at instants $k$ and $k+1$, the system's states deviate from the switching plane. The range of these deviations depends not only on the dynamical properties of the system but also on the disturbance magnitudes and the choice of the discretization period $[9,10]$. This behaviour causes rising magnitudes of the control signal, and as a result, the representative point of the system begins to chatter around the sliding plane. In order to avoid these problems, it is necessary to transfer the sliding mode control design to discrete time domain, resulting in a quasisliding mode. 
The study of the discretization effects in the sliding mode began in the 1980s. As a precursor of the idea, Milosavljević defined the quasi-sliding mode as a motion of the system's representative point within a band around the sliding plane, not on the plane itself [11]. The conditions of existence and stability of such quasi-sliding mode were further discussed in [12-14]. Once the properties of the quasi-sliding mode have been stated, various authors proposed different control design methods. Furuta in $[15,16]$ derived the sliding mode control law from the stability analysis with a Lyapunov function utilizing the sliding variable $s(k)$. On the other hand, Utkin et al. $[17,18]$ considered the stability analysis separately and proposed a control that would drive the system's states onto the sliding plane in one step by assigning $s(k+1)=0$. However, the most popular has become the reaching law approach introduced by Gao et al. [19]. The authors decided to drive the sliding variable $s(k)$ to the sliding plane in a designed number of steps with an application of a reaching law. According to their study, once the representative point of the system enters a specified band around the plane [20], it continues in a zigzag motion along it. The reaching law approach demonstrates relative law complexity, while ensuring a reduction of the control effort in comparison to Utkin's method and providing a specified ultimate band width. Therefore, it has been an inspiration for countless later publications [21-30]. Some authors modified Gao's reaching law [21-24]; others proposed new reaching functions [25-30]. The latest trends in discrete time sliding mode are multirate output feedback $[31,32]$, event triggered sliding mode [33], or higher relative degree outputs [34-37].

This study takes into account a sampled data system, presents the control design, and discusses the influence of the choice of the discretization period $T$ on the properties of the achieved quasi-sliding mode. We propose a new reference sliding variable-based control scheme. Inspired by the reaching law of Gao et al. [19], we consider two types of the reference sliding variable generation, adapting differently to changes of the discretization period. Further, we implement a reference sliding variable following reaching law for a real disturbed system. In order to improve its robustness, we eliminate the chattering problem with the application of the nonswitching type reaching law and incorporate a disturbance compensation algorithm, which reduces the ultimate band width and the maximum errors of all state variables to $O\left(T^{2}\right)$ order.

The key idea of the reference sliding variable based control is to reduce the disturbance influence on the system. All conventional sliding mode reaching laws [19, 21-30] use the current value of the sliding variable $s(k)$ to obtain the control signal for the next step $k+1$. As the system is under the influence of external disturbances, their effects are accumulated in the value of the sliding variable through the whole control process. Consequently, these disturbances are transferred to the control signal value. In other words, the control is calculated from the disturbed data. However, the reference sliding variable-based control proposed in this study avoids this problem. Unlike other mentioned publications, this paper assumes controlling the plant according to a predefined reference sliding variable profile. Therefore, the control signal calculation is based on the undisturbed reference sliding variable value instead of the actual plant's sliding variable. Consequently, the sliding variable $s(k+1)$ is influenced by the disturbance from one previous step $D(k)$, whereas in the classical control methods, it is influenced by $D(0), D(1), \ldots, D(k)$. As a result, the robustness of the system is significantly improved. Furthermore, the proposed control strategy uses a disturbance compensation algorithm [28-30], which ensures the reduction of the achieved quasisliding mode band width to $O\left(T^{2}\right)$ order. Finally, unlike other publications, this study also examines the errors of the state variables of the system, and the maximum error values for all state variables are derived. As the closed-loop system's robustness depends directly on the choice of the discretization period, we present and compare two different methods of the reference sliding variable generation, which adapt differently to changes of the discretization period. One ensures constant convergence pace against continuous time and the other provides constant convergence pace against the number of discrete time instants.

The paper is organized in the following way. In Section 2, we describe the continuous time dynamical problem and carry out the discretization procedures. Next, we summarize the seminal discrete time sliding mode control method introduced by Gao et al. [19]. Section 4, step by step, describes the design of our new control algorithm and presents its properties. Finally, we present our simulation results, and Section 5 includes some final remarks and conclusions.

\section{Dynamical System}

We consider a continuous time disturbed system:

$$
\dot{\mathbf{x}}(t)=\mathbf{A x}(t)+\mathbf{b}[u(t)+f(t)]
$$

where $\mathbf{x}(t)$ is the $n \times 1$ state vector, $u(t)$ is a digitally implemented control input, $f(t)$ is the disturbance, and $\mathbf{A}$ and $\mathbf{b}$ are of appropriate dimensions. The disturbance $f(t)$ is a smooth time function restricted by

$$
|f(t)| \leq f_{\max }
$$

which makes the control feasible. The aim of the control process is to drive the system from the initial position $\mathbf{x}(0)=$ $\mathbf{x}_{\mathbf{0}}$ to zero. We assume that the system operates with sampled data, as the state measurements and the control updates take place at discrete time instants only. This enforces transferring the analysis to the discrete time domain. For that purpose, we discretize system (1) with the discretization period $T$ obtaining: 


$$
\boldsymbol{\eta}(k+1)=\boldsymbol{\Phi} \boldsymbol{\eta}(k)+\Gamma u(k)+\mathbf{d}(k),
$$

where the state matrix $\Phi$ and the input distribution vector $\Gamma$ are calculated as follows:

$$
\begin{aligned}
& \boldsymbol{\Phi}=e^{\mathbf{A} T}, \\
& \Gamma=\int_{0}^{T} e^{\mathbf{A} \tau} \mathrm{d} \tau \mathbf{b} .
\end{aligned}
$$

From (4), we notice that in the discrete time domain, the order of the input distribution vector $\Gamma$ linearly depends on the discretization period $T$. Lastly, we calculate the discretized disturbance vector $\mathbf{d}(k)$ as

$$
\mathbf{d}(k)=\int_{0}^{T} e^{\mathbf{A} \tau} \mathbf{b} f[(k+1) T-\tau] \mathrm{d} \tau .
$$

Considering (4) and (5), we conclude that the discrete time system's description depends on the choice of the discretization period $T$. Integrating (4) and (5), the input vector $\boldsymbol{\Gamma}$ and the disturbance vector $\mathbf{d}(k)$ are both of $O(T)$ order. Consequently, the effects of any changes of $T$ on the controller's properties will be studied further in this paper.

\section{Conventional Control Scheme}

3.1. Choice of the Sliding Plane. The aim of our study is to design a sliding mode controller for the sampled data system (1) discretized to (3). Controller's task is to drive all state variables $\eta_{1}, \eta_{2}, \ldots, \eta_{n}$ to zeros. Therefore, we incorporate the values of the state variables into a sliding variable $\mathbf{s}(k)=$ $\mathbf{c} \boldsymbol{\eta}(k)$, where vector $\boldsymbol{c}$ ensures that $\mathbf{c} \boldsymbol{\Gamma} \neq 0$. As the choice of $\mathbf{c}$ is arbitrary, we assume that $\mathbf{c}=\left[c_{1}, c_{2}, \ldots, c_{n-1}, 1\right]$, so the vector is of $O$ (1) order. The controller must drive the representative point of the system from $s(0)=\mathbf{c} \eta(0)$ to the sliding plane:

$$
s(k+1)=0 .
$$

In this paper, we choose the sliding plane in a dead-beat manner so that when $s(k)=0$, the system's characteristic polynomial satisfies

$$
M(z)=\operatorname{det}\left(1_{n \times n} z-\mathbf{N}\right)=z^{n},
$$

where $\mathbf{N}=\left[\mathbf{1}_{n \times n}-\boldsymbol{\Gamma}(\mathbf{c} \boldsymbol{\Gamma})^{-1} \mathbf{c}\right] \boldsymbol{\Phi}$ represents the state matrix of the closed-loop system. This specific choice of the sliding plane guarantees stability of the closed-loop system, as all its poles are placed at the origin of the complex plane. Moreover, with this selection of $\mathbf{c}$, the state matrix $\mathbf{N}$ becomes nilpotent, so $\mathbf{N}^{n}=0$. This results in some favourable properties utilized further in this paper.

In order to compensate for the disturbance $\mathbf{d}$ $(k)$, we denote its impact on the value of the sliding variable with

$$
D(k)=\mathbf{c d}(k) \text {. }
$$

Considering that the continuous time disturbance is lower and upper bounded by $\pm f_{\max }$, we conclude that $D(k)$ is bounded as well and satisfies

$$
|D(k)|=|\mathbf{c d}(k)| \leq D_{\max },
$$

where $D_{\max }$, as well as $\mathbf{d}(k)$, is of $O(T)$ order, while $\mathbf{c}$ is of order $O(1)$.

3.2. Seminal Reaching Law of Gao Et Al. In 1995, Gao et al. [19] proposed a fundamental sliding mode control strategy for system (3). The authors noticed that demanding the system to reach the sliding plane in one control step:

$$
s(k+1)=0,
$$

as previously studied in $[17,18]$, may require unnecessarily large control values. It may also result in undesirable values of the state variables during the control process. Therefore, Gao et al. [19] suggested to use the following reaching law to control the convergence pace:

$$
s(k+1)=(1-q T) s(k)-\varepsilon T \operatorname{sgn}[s(k)]+D(k) .
$$

The parameters of (11) must satisfy

$$
0<q T<1, \quad \varepsilon>0 .
$$

The reaching function (11) consists of a proportional term with factor $q T$ and a switching term with factor $\varepsilon T$. Consequently, the reaching law forces the representative point of the system to cross the sliding plane in each successive time step in the sliding phase. However, the switching type definition of the quasi-sliding mode turns out to be impractical, as it leads to chattering. Therefore, multiple studies [22-24] considered elimination of the signum function from (11) which gives the following reaching law:

$$
s(k+1)=(1-q T) s(k)+D(k) .
$$
signal

The application of (13) for system (3) requires the control

$$
u(k)=-(\mathbf{c} \Gamma)^{-1}\{\mathbf{c} \Phi \mathbf{\eta}(k)-(1-q T) s(k)\} .
$$

The proportional term $(1-q T) s(k)$ ensures that the value of the sliding variable is reduced in each step until $q T \mid s$ $(k) \mid$ becomes smaller than the disturbance $|D(k)|$. Taking into account (9), we conclude that the representative point of the system approaches the sliding plane monotonically until it reaches the quasi-steady state when

$$
|s(k)|<\frac{D_{\max }}{q T} .
$$

Once the representative point reaches the band (15) it never leaves it again. As $D_{\max }$ is of $O(T)$ order, the achieved quasisliding mode band width is of $O(1)$ order, so it does not depend on the choice of the discretization period. Appealing as it may seem, after further study, we notice that this is a clear 
disadvantage of Gao's strategy. As we decrease the discretization period, the continuous time system (1) becomes exposed to the disturbance $f(t)$ for shorter periods of time between the control updates. That shall result in a reduction of the quasi-sliding mode band width. However, the proportional factor $q$ is also scaled by the discretization period $T$. Therefore, with the reduction of $T$, the pace of convergence is reduced as well. In other words, the reaching law of Gao is designed to maintain constant pace of convergence against time, not against the number of discrete time instants. In the next section, we will propose a new control strategy using a reference sliding variable profile. We will consider and compare two versions of Gao's reaching law. The first one ensures constant convergence pace against time and the second one ensures constant convergence pace against the number of discrete time instants. We will prove that application of the digital controller for system (1) may have positive impact on the system's robustness.

\section{Reference Sliding Variable following SMC}

In this section, we propose an innovative control strategy based on an external reference sliding variable generator. The key of our control scheme is to generate the desired evolution for the sliding variable externally, utilizing the system's mathematical model and a nonswitching type reaching law. Next, we incorporate the reference sliding variable into the reaching law for the real system (3). We aim to drive the plant's sliding variable to the desired value with accuracy of the disturbance. Consequently, the control becomes dependant only on the last value of the disturbance $D$ $(k)$. As the reference profile determines the pace of convergence and the width of the quasi-sliding mode band, the initial conditions must be set the same as for the system and the control parameters must ensure the demanded behaviour. The proposed structure of the closed-loop system is depicted in the following block diagram (Figure 1).

4.1. Constant Convergence Pace against Time. We begin with presenting two methods for generation of the demand sliding variable evolution. The reference profile must not only ensure all the properties of the quasi-sliding mode but also eliminate the chattering problem. Therefore, we adapt the following nonswitching type definition of the quasi-sliding mode. We assume that the quasi-sliding mode exists when the representative point of the system approaches the sliding plane monotonically, and once it enters a specified band around the sliding plane, it will never exceed it again. However, it is not demanded that the sliding variable changes its sign. In order to ensure this kind of motion, we use the following reaching law:

$$
s_{r}(k+1)=(1-q T) s_{r}(k),
$$

where $s_{r}(k)$ represents the reference sliding variable in step $k$ and $0<q T<1$. Considering that the reference profile must demonstrate the desired trajectory of the system, we set the initial condition as

$$
s_{r}(0)=s(0)=s_{0}
$$

As the reference sliding variable profile is not influenced by any external disturbances, elimination of the switching term from the reaching law ensures that, for any $k \geq 0$, we get

$$
\operatorname{sgn}\left[s_{r}(k+1)\right]=\operatorname{sgn}\left[s_{r}(k)\right]=\operatorname{sgn}\left(s_{0}\right),
$$

so no chattering appears. Function (16) also implies that the value of the reference sliding variable is in each step reduced by a proportional term $q T s_{r}(k)$. Consequently, any changes of the discretization period $T$ result in changes of the pace of convergence. Any reduction of $T$ results in slower convergence to the sliding plane. We may say that with constant choice of $q$, this reaching law ensures that the system converges to the sliding plane in the same time, not in the same number of discrete time instants, regardless of the discretization period of the controller. Once $s_{r}(k)$ reaches the quasi-steady state from (16), we get

$$
s_{r}(k+1)=(1-q T) s_{r}(k)=s_{r}(k)=0 .
$$

Therefore, in the sliding phase, the reference sliding variable remains on the sliding plane.

4.2. Constant Convergence Pace against Discrete Instants. The second version of our control algorithm is designed to provide convergence to the sliding plane in a constant number of steps, regardless of any changes of the discretization period. For that purpose, we eliminate $T$ from the proportional term obtaining a reaching law in the form

$$
s_{r}(k+1)=(1-q) s_{r}(k)
$$

where $0<q<1$. The initial condition remains as defined in (17). As (20) does not include any switching element, (18) holds. In the quasi-steady state, we get

$$
s_{r}(k+1)=(1-q) s_{r}(k)=s_{r}(k),
$$

which implies that when $k$ tends to infinity, $s_{r}(k)$ tends to zero. However, in this case, the pace of convergence of the system does not depend on the discretization period $T$. The value of $s_{r}(k)$ is in each step reduced by the proportional term $q s_{r}(k)$. Therefore, knowing the initial condition $s_{0}$, we may select the value of $q$ to drive the system to the sliding plane in a selected number of steps. In other words, for the constant value of the proportional factor $q, s_{r}(k)$ reaches zero after the same number of discrete time instants regardless of the discretization period. Unlike reaching law (16), which ensured constant convergence pace against continuous time, this version of the reaching law results in a constant convergence pace against the number of discrete time instants. Therefore, with the reduction of the discretization period, (21) results in faster reaction of the closed-loop system, as $s_{r}(k)$ reaches zero in shorter time.

4.3. Reference Sliding Variable-Based Reaching Law. Once we have obtained the desired profile of the sliding variable $s_{r}(k)$, we now present the new control law for the real disturbed system (3). We apply a reference sliding variable following reaching law: 


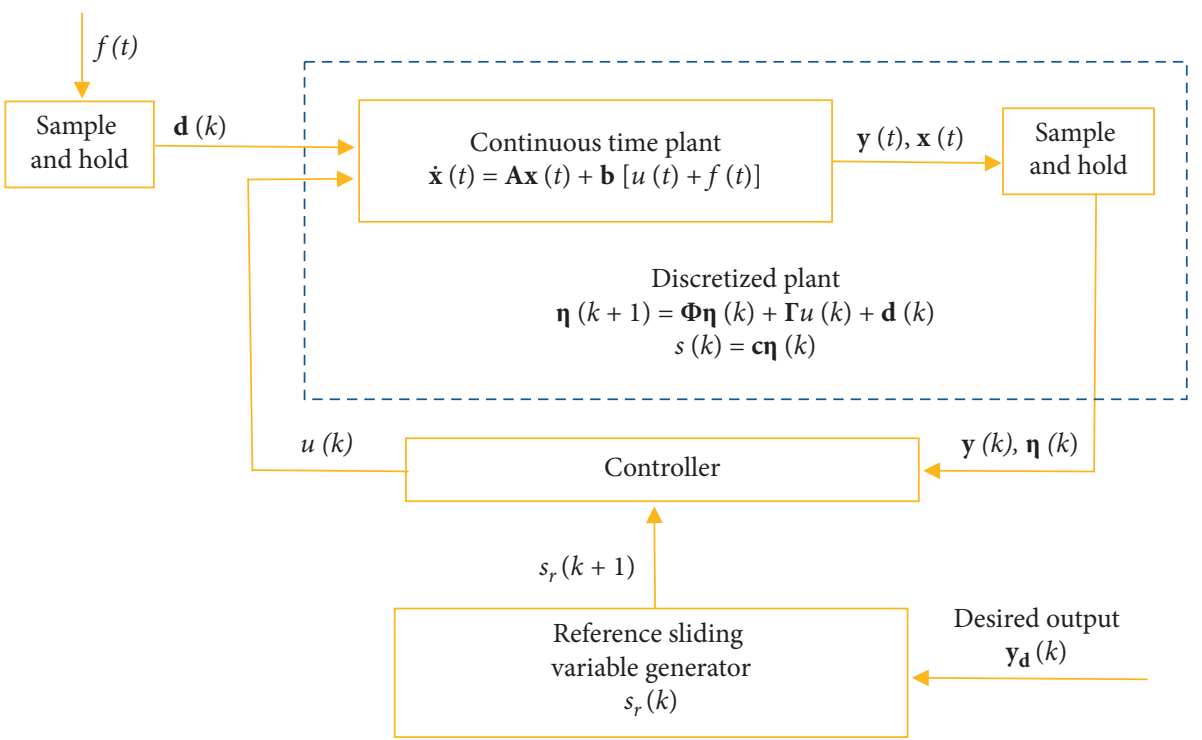

Figure 1: Block diagram of the proposed closed-loop system.

$$
s(k+1)=s_{r}(k+1)+D(k) .
$$

Reaching law (22) is designed to drive the plant's sliding variable to the reference value with accuracy of the disturbance $D(k)$. From (22), (3), and (6), we calculate the control as

$$
u(k)=-(\mathbf{c} \Gamma)^{-1}\left\{\mathbf{c} \Phi \boldsymbol{\eta}(k)-s_{r}(k+1)\right\} .
$$

Taking into account the restriction of the disturbance influence (9), from (22), we get

$$
|s(k+1)| \leq\left|s_{r}(k+1)\right|+D_{\max } .
$$

Regardless of the chosen method for generation of the reference sliding variable, in the previous sections we have shown that in the quasi-steady state, $s_{r}(k)=0$. This observation leads to the following restriction of the quasi-sliding mode band width:

$$
|s(k)| \leq D_{\max } .
$$

Considering that $D_{\max }$ is of $O(T)$ order, we conclude that the obtained ultimate band width linearly depends on the discretization period. Therefore, the width of the band decreases with the reduction of $T$. As in Gao's control law $q T<1$, comparing (15) and (25), one may easily notice that the reference sliding variable-based control ensures an improvement of the system's robustness.

Reaching law (22) also limits the impact of disturbances on the sliding variable to one control step. However, the disturbance influence may be further reduced with application of a disturbance compensation algorithm. As (22) provides the accuracy of the disturbance $D(k)$, we may express the control error as

$$
s(k+1)-s_{r}(k+1)=D(k) .
$$

Therefore, the sum of the control errors from all previous steps $0,1, \ldots, k$, will compensate for the disturbance value $D$ $(k-1)$. Consequently, we introduce the following modification [25] to the reaching law:

$$
s(k+1)=s_{r}(k+1)+D(k)-\sum_{l=0}^{k}\left[s(l)-s_{r}(l)\right] .
$$

From (3), (6), and (27), we obtain the control law:

$$
u(k)=-(\mathbf{c} \Gamma)^{-1}\left\{\mathbf{c} \Phi \mathbf{\eta}(k)-s_{r}(k+1)+\sum_{l=0}^{k}\left[s(l)-s_{r}(l)\right]\right\} \text {. }
$$

One may notice from (27) that the disturbance compensation term introduces additional dynamics to the closedloop system, which enforces stability analysis. We may see that the sum on the right-hand side of (27) represents

$$
\sum_{l=0}^{k}\left[s(l)-s_{r}(l)\right]=s(k)-s_{r}(k)+\sum_{l=0}^{k-1}\left[s(l)-s_{r}(l)\right] .
$$

Using (27) for $s(k)$ again, we get

$$
\sum_{l=0}^{k}\left[s(l)-s_{r}(l)\right]=D(k-1)
$$

From (30), one may directly see that the disturbance compensation term in step $k$ represents the disturbance $D$ $(k-1)$. Therefore, the current disturbance value $D(k)$ is compensated with its previous value $D(k-1)$. Regardless of the chosen reference sliding variable generation method, in the sliding phase, when $s_{r}(k+1)=0$, reaching law (27) becomes

$$
s(k+1)=D(k)-D(k-1) .
$$

Consequently, the stability of the disturbance estimation must be analysed. With application of the disturbance compensator, in the sliding phase, the closed-loop system is described with the previous state vector $\boldsymbol{\eta}$ and the dynamical control signal $u$, which results in the following set of state equations: 


$$
\begin{aligned}
& \boldsymbol{\eta}(k+1)=\boldsymbol{\Phi} \boldsymbol{\eta}(k)+\Gamma u(k)+\mathbf{d}(k-1), \\
& u(k+1)=-(\mathbf{c} \Gamma)^{-1}[\mathbf{c} \boldsymbol{\Phi} \boldsymbol{\eta}(k+1)+\mathbf{c d}(k-1)],
\end{aligned}
$$

and in a new state vector $[\boldsymbol{\eta}(k) u(k)]^{T}$ of $n+1$ length. Substituting for $\boldsymbol{\eta}(k+1)$, we get the control signal:

$$
\begin{aligned}
u(k+1)= & -(\mathbf{c} \Gamma)^{-1}\left[\mathbf{c} \Phi^{2} \boldsymbol{\eta}(k)+\mathbf{c} \Phi \Gamma u(k)\right. \\
& \left.+\mathbf{c}\left(\Phi+1_{n \times n}\right) \mathbf{d}(k-1)\right] .
\end{aligned}
$$

Implementing control (33) into (32) results in the following state matrix of the closed-loop system:

$$
\widehat{\boldsymbol{\Phi}}=\left[\begin{array}{cc}
\Phi & \Gamma \\
-(\mathrm{c} \Gamma)^{-1} \mathbf{c} \Phi^{2} & -(\mathrm{c} \Gamma)^{-1} \mathbf{c} \Phi \Gamma
\end{array}\right],
$$

and the following state equation:

$$
\left[\begin{array}{l}
\boldsymbol{\eta}(k+1) \\
u(k+1)
\end{array}\right]=\widehat{\boldsymbol{\Phi}}\left[\begin{array}{l}
\boldsymbol{\eta}(k) \\
u(k)
\end{array}\right]+\left[\begin{array}{c}
1_{n \times n} \\
-(\mathbf{c} \Gamma)^{-1} \mathbf{c}\left(\boldsymbol{\Phi}+1_{n \times n}\right)
\end{array}\right] \mathbf{d}(k-1) .
$$

In order to determine the stability of the system, we calculate its characteristic polynomial:

$$
\begin{aligned}
M(z) & =\operatorname{det}\left(1_{(n+1) \times(n+1)} z-\widehat{\boldsymbol{\Phi}}\right) \\
& =\left|\begin{array}{cc}
1_{n \times n} z-\boldsymbol{\Phi} & -\Gamma \\
(\mathrm{c} \Gamma)^{-1} \mathbf{c} \Phi^{2} & z+(\mathrm{c} \Gamma)^{-1} \mathbf{c} \boldsymbol{\Phi} \Gamma
\end{array}\right| .
\end{aligned}
$$

The eigenvalues of (36) may be easily found using the following similarity transformation:

$$
\widehat{\boldsymbol{\Phi}}=\left[\begin{array}{cc}
\boldsymbol{\Phi} & \Gamma \\
-(\mathbf{c} \Gamma)^{-1} \mathbf{c} \boldsymbol{\Phi}^{2} & -(\mathbf{c} \Gamma)^{-1} \mathbf{c} \boldsymbol{\Phi} \Gamma
\end{array}\right]=\mathbf{M}^{-1}\left[\begin{array}{cc}
\mathbf{N} & \Gamma \\
0_{1 \times n} & 0
\end{array}\right] \mathbf{M},
$$

where $\mathbf{N}=\left[\mathbf{1}_{n \times n}-\boldsymbol{\Gamma}(\mathbf{c} \boldsymbol{\Gamma})^{-1} \mathbf{c}\right] \boldsymbol{\Phi}$ represents the state matrix of the system without the disturbance compensator and

$$
\mathbf{M}=\left[\begin{array}{cc}
1_{n \times n} & 0_{n \times 1} \\
(\mathbf{c} \Gamma)^{-1} \mathbf{c} \Phi & 1
\end{array}\right]
$$

From (37), it may be concluded that $n$ eigenvalues of the system remain the same as in the case without the disturbance compensator and may be determined from $z_{i}=$ eig $\left\{\left[\mathbf{1}_{n \times n}-\boldsymbol{\Gamma}(\mathbf{c} \boldsymbol{\Gamma})^{-1} \mathbf{c}\right] \boldsymbol{\Phi}\right\}$, for $i=1, \ldots, n$. The disturbance compensation term is stable, as it brings one additional eigenvalue $z_{n+1}=0$. Consequently, the closed-loop system's stability depends on the selection of control vector $\mathbf{c}$ only. Next, we will prove that the proposed control strategy results in the quasi-sliding mode band width of $O\left(T^{2}\right)$ order.

Theorem 1. Reaching law (27) guarantees monotonic convergence of the representative point of system (3) to a band of $O\left(T^{2}\right)$ width around the sliding plane (6).

Proof. To prove the above, we first use observation (30) to simplify the reaching law to the following form:

$$
s(k+1)=s_{r}(k+1)+D(k)-D(k-1) .
$$

Various authors have studied the nature of discretized disturbance $\mathbf{d}(k)$. In [28], it has been noticed that the Taylor series expansion may be used to approximate the disturbance values in discrete time domain. Following that study, d $(k)$ may be expressed as

$$
\begin{aligned}
\mathbf{d}(k)= & \int_{0}^{T} e^{\mathbf{A} \tau} \mathbf{b} f(k T) \mathrm{d} \tau+\int_{0}^{T} e^{\mathbf{A} \tau} \mathbf{b} v(k T)(T-\tau) \mathrm{d} \tau \\
& +\int_{0}^{T} e^{\mathbf{A} \tau} \mathbf{b} \frac{1}{2 !} w(\mu)(T-\tau)^{2} \mathrm{~d} \tau
\end{aligned}
$$

where $v(t)$ and $w(t)$ represent the first and second derivative of $f(t)$, respectively, and $\mu$ is an instant between $k T$ and $(k+1) T$. After some further transformations, we get

$$
\mathbf{d}(k)=\Gamma f(k T)+\frac{1}{2} \Gamma v(k T) T+O\left(T^{3}\right) .
$$

As visible from (41), d $(k)$ includes a matched term, whose range depends on the control vector $\Gamma$. As $\Gamma$ is of $O(T)$ order, $\mathbf{d}(k)$ depends on $T$ linearly as well. The discretized disturbance also contains a mismatched component of $O$ $\left(T^{3}\right)$ order. Moreover, the change of $\mathbf{d}(k)$ between two consecutive time steps also depends on the discretization period in the following relation:

$$
\mathbf{d}(k)-\mathbf{d}(k-1)=O\left(T^{2}\right),
$$

which has been proved in $[10,28]$. This implies that the rate of change of $D(k)$ is restricted and becomes of $O\left(T^{2}\right)$ order. Consequently, for $D(k)-D(k-1)$, we may write

$$
\alpha=\max \{|c[d(k)-d(k-1)]|\}=O\left(T^{2}\right),
$$

where $\alpha$ denotes the maximum rate of change. Therefore, for any $k \geq 0, D(k)$ satisfies

$$
|D(k)-D(k-1)| \leq \alpha .
$$

Coming back to reaching law (39), we notice that regardless of the method of generation of $s_{r}(k)$, in the quasisliding phase, it holds that

$$
s_{r}(k)=0 .
$$

Taking into account (45) and (30), in the quasi-steady state, from (39), we get

$$
s(k+1)=D(k)-\sum_{l=0}^{k}\left[s(l)-s_{r}(l)\right]
$$

which describes the evolution of the sliding variable in the sliding phase. It may be concluded from (46) that the width of the quasi-sliding mode band depends on the rate of change of $D(k)$. Therefore, considering (30) and (44), we notice that in the sliding phase, the representative point of the system remains within a band restricted as follows:

$$
|s(k)| \leq \alpha=O\left(T^{2}\right),
$$

which ends the proof.

As visible directly from (47), the application of the disturbance compensator results in a band depending on 
the discretization period $T$ in a quadratic relation. Consequently, considering that $D_{\max }$ is of $O(T)$ order, from the comparison of (15), (25), and (47), for appropriate values of $T$, we may write

$$
\alpha<D_{\max }<\frac{D_{\max }}{q T} .
$$

It is clear from (48) that our new control strategy ensures a significant reduction of the quasi-sliding mode band width, which results in an improvement of the system's robustness. It is also worth noticing that our control law (28) does not impose any switching condition on the sliding variable. Therefore, chattering is reduced to minimum, as it only appears when enforced by the uncompensated disturbance term.

4.4. State Error Estimation. We have shown that the proposed control strategy results in the ultimate band width of $O\left(T^{2}\right)$ order. Next, we will show that restriction of the value of the sliding variable results in a restriction of the values of state variables of the system. Therefore, in the quasi-steady state, all state variable errors become of $O\left(T^{2}\right)$ order as well. For the sake of clarity of our calculations, considering (41), we may express $\mathbf{d}(k)$ as the sum of matched disturbance effects $\Gamma \sigma(k)$ and the mismatched term of $O\left(T^{3}\right)$ order:

$$
\mathbf{d}(k)=\Gamma \sigma(k)+O\left(T^{3}\right) .
$$

Theorem 2. If vector $c$ is chosen so that (7) holds and system (3) is controlled according to (28), then the state variables satisfy

$$
\eta_{i}(j)=\sum_{g=0}^{n-1} \boldsymbol{\delta}_{\mathbf{i}} \mathbf{N}^{g} \Gamma(\mathbf{c} \Gamma)^{-1} s(j-g)+O\left(T^{3}\right),
$$

where $j \geq n$ and $\boldsymbol{\delta}_{\boldsymbol{i}}$ is a row vector such that

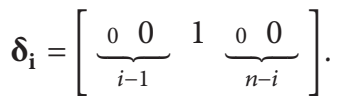

Proof. In any moment $k+1$, we may express the state vector using (3) and (28) as

$$
\begin{aligned}
\boldsymbol{\eta}(k+1)= & \boldsymbol{\Phi} \boldsymbol{\eta}(k)-\Gamma(\mathbf{c} \Gamma)^{-1} \mathbf{c} \boldsymbol{\Phi} \boldsymbol{\eta}(k)+\Gamma(\mathbf{c} \Gamma)^{-1} s_{r}(k+1) \\
& -\Gamma(\mathbf{c} \Gamma)^{-1} \sum_{l=0}^{k}\left[s(l)-s_{r}(l)\right]+\mathbf{d}(k) .
\end{aligned}
$$

Considering that the closed-loop state matrix is denoted with $\mathbf{N}$ and taking into account (30), we transform (52) to the shortened form:

$$
\begin{aligned}
\boldsymbol{\eta}(k+1)= & \mathbf{N} \boldsymbol{\eta}(k)+\Gamma(\mathbf{c} \Gamma)^{-1} s_{r}(k+1) \\
& -\Gamma(\mathbf{c} \Gamma)^{-1} D(k-1)+\mathbf{d}(k) .
\end{aligned}
$$
get

Following the same routine, we continue for $\boldsymbol{\eta}(k+2)$ to

$$
\begin{aligned}
\boldsymbol{\eta}(k+2)= & \mathbf{N}^{2} \boldsymbol{\eta}(k)+\mathbf{N} \Gamma(\mathbf{c} \Gamma)^{-1} s_{r}(k+1) \\
& -\mathbf{N} \Gamma(\mathbf{c} \Gamma)^{-1} D(k-1)+\mathbf{N} \mathbf{d}(k) \\
& +\Gamma(\mathbf{c} \Gamma)^{-1} s_{r}(k+2)-\Gamma(\mathbf{c} \Gamma)^{-1} D(k)+\mathbf{d}(k+1) .
\end{aligned}
$$
by

Finally, for any $j \geq 0$, the state vector $\boldsymbol{\eta}(j)$ is represented

$$
\begin{aligned}
\boldsymbol{\eta}(j)= & \mathbf{N}^{j-k} \boldsymbol{\eta}(k)+\sum_{g=0}^{j-k-1} \mathbf{N}^{g}\left[\Gamma(\mathbf{c} \Gamma)^{-1} s_{r}(j-g)\right. \\
& \left.-\Gamma(\mathbf{c} \Gamma)^{-1} D(j-g-2)+\mathbf{d}(j-g-1)\right] .
\end{aligned}
$$

Using the fact that the new state matrix $\mathbf{N}$ is nilpotent, for any $j-k \geq n$, we get $\mathbf{N}^{j-k}=0$. Therefore, for any $j \geq k+n$, (55) may be shortened to

$$
\begin{aligned}
\boldsymbol{\eta}(j)= & \sum_{g=0}^{n-1} \mathbf{N}^{g}\left[\Gamma(\mathbf{c} \Gamma)^{-1} s_{r}(j-g)-\Gamma(\mathbf{c} \Gamma)^{-1} D(j-g-2)\right. \\
& +\mathbf{d}(j-g-1)] .
\end{aligned}
$$

On the other hand, using (49), we may write

$$
\Gamma(\mathbf{c} \Gamma)^{-1} D(k)=\Gamma(\mathbf{c} \Gamma)^{-1} \mathbf{c}\left[\Gamma \sigma(k)+O\left(T^{3}\right)\right]=\Gamma \sigma(k)+O\left(T^{3}\right) .
$$

Considering (27) and (57), one may notice that

$$
\begin{aligned}
& \Gamma(\mathbf{c} \Gamma)^{-1} s_{r}(j-g)-\Gamma(\mathbf{c} \Gamma)^{-1} D(j-g-2)+\mathbf{d}(j-g-1) \\
& \quad=\Gamma(\mathbf{c} \Gamma)^{-1} s(j-g)+O\left(T^{3}\right) .
\end{aligned}
$$

Using (58), we simplify (56) to

$$
\boldsymbol{\eta}(j)=\sum_{g=0}^{n-1} \mathbf{N}^{g} \Gamma(\mathbf{c} \Gamma)^{-1} s(j-g)+O\left(T^{3}\right) .
$$

Next, by multiplying both sides of (59) by the row vector $\boldsymbol{\delta}_{\mathbf{i}}$, we get the $i$-th state variable expressed as

$$
\eta_{i}(j)=\sum_{g=0}^{n-1} \boldsymbol{\delta}_{\mathbf{i}} \mathbf{N}^{g} \Gamma(\mathbf{c} \Gamma)^{-1} s(j-g)+O\left(T^{3}\right),
$$

which ends the proof.

We also notice that in the sliding phase, the sliding variable is limited by $\alpha$. Consequently, in the sliding phase, all the state variables are limited by

$$
\left|\eta_{i}(j)\right| \leq \alpha \sum_{g=0}^{n-1}\left|\boldsymbol{\delta}_{\mathbf{i}} \mathbf{N}^{g} \Gamma(\mathbf{c} \Gamma)^{-1}\right|+O\left(T^{3}\right)
$$

As $\alpha$ is of $O\left(T^{2}\right)$ order, the state variables are confined to the band of $O\left(T^{2}\right)$ width as well. On the other hand, in the original control strategy, with reaching law (13), the quasisliding mode band is of $2 D_{\max } / q T$ width. As the width of this band is of $O(1)$ order, the state variables are limited to $O(1)$ 
order too. Therefore, we have shown that the proposed control law not only reduces the width of the ultimate band but also reduces the errors of all state variables.

\section{Simulation Example}

In this section, we present a simulation example to demonstrate the properties of our new control method. We consider a second-order continuous time system:

$$
\dot{x}(t)=\left[\begin{array}{cc}
0 & 1 \\
0 & -0.75
\end{array}\right] \mathbf{x}(t)+\left[\begin{array}{l}
0 \\
1
\end{array}\right][u(t)+f(t)]
$$

where the initial conditions are $\mathbf{x}_{0}=\mathbf{x}(0)=[10-1]^{T}$. Such a plant may for example represent a simple mechanical actuator. The disturbance $f(t)$ is a sinusoidal function with amplitude of 1 and frequency of $0.5 \mathrm{~Hz}$, so $f_{\max }=1$. The system operates on sampled data. Therefore, we need to design a discrete time sliding mode controller to drive all the state variable to zeros. We would like to show the impact of the chosen discretization period on the system's performance. Therefore, we present three cases with discretization periods: $T=0.1 \mathrm{~s}, 0.05 \mathrm{~s}$, and $0.01 \mathrm{~s}$. For all three cases, we select the sliding variable as $s(k)=\mathbf{c} \eta(k)$ and the sliding plane $s(k+1)=0$. Vector $c$ is chosen for each case separately so that (7) always holds. To compare the pace of convergence of the systems, we set the value of $q=3$ for the proportional factor $q T$, which gives $q T=0.3,0.15$, and 0.03 for $T=0.1 \mathrm{~s}$, $0.05 \mathrm{~s}$, and $0.01 \mathrm{~s}$, respectively, and 0.3 , where the proportional factor has a constant value of $q$. We compare the effectiveness of our control method to the nonswitching type control of Gao (14).

We must begin the design of the controller with obtaining the appropriate discrete time system representation. The discretization process led us to the following discrete time descriptions. Firstly, for $T=0.1 \mathrm{~s}$, we obtained

$$
\boldsymbol{\eta}(k+1)=\left[\begin{array}{cc}
1 & 0.09634 \\
0 & 0.9277
\end{array}\right] \boldsymbol{\eta}(k)+\left[\begin{array}{c}
0.004877 \\
0.09634
\end{array}\right] u(k)+\mathbf{d}(k) \text {. }
$$

According to (7), we set $\mathbf{c}=[20.25221]$ so that all poles of the closed-loop system are equal to zero. Having the discrete time system's representation, we calculate the maximum disturbance impact:

$$
D_{\max }=0.1951=1.951 \cdot T,
$$

which gives $D_{\max } / q T=0.65$. For $T=0.1 \mathrm{~s}$, the continuous time disturbance $f(t)$ changes maximally by 0.314 , which results in

$$
\alpha=0.0613=6.13 \cdot 10^{-2}=6.13 \cdot T^{2} .
$$

Next, we consider system (62) discretized with $T=0.05 \mathrm{~s}$. In this case, we obtain

$$
\boldsymbol{\eta}(k+1)=\left[\begin{array}{cc}
1 & 0.04907 \\
0 & 0.9632
\end{array}\right] \boldsymbol{\eta}(k)+\left[\begin{array}{c}
0.001235 \\
0.04907
\end{array}\right] u(k)+\mathbf{d}(k) .
$$

In order to satisfy (7), we set $\mathbf{c}=\left[\begin{array}{l}40.277 \\ 1\end{array}\right]$. Hence,

$$
D_{\max }=0.0978=1.956 \cdot T \text {, }
$$

and $D_{\max } / q T=0.65$. The maximum change of $f(t)$ between two consecutive time steps is 0.157 , which gives

$$
\alpha=0.0155=1.55 \cdot 10^{-2}=6.2 \cdot T^{2} .
$$

Finally, we discretize system (62) with $T=0.01 \mathrm{~s}$ obtaining

$$
\boldsymbol{\eta}(k+1)=\left[\begin{array}{cc}
1 & 0.009963 \\
0 & 0.9925
\end{array}\right] \boldsymbol{\eta}(k)+\left[\begin{array}{c}
0.00005 \\
0.009963
\end{array}\right] u(k)+\mathbf{d}(k) .
$$

In this case, $\mathbf{c}=\left[\begin{array}{ll}200.7198 & 1\end{array}\right]$.

$$
D_{\max }=0.01958=1.958 \cdot T,
$$

and $D_{\max } / q T=0.65$. The maximum rate of change of $f(t)$ is 0.0314. Therefore,

$$
\alpha=0.00062=6.2 \cdot T^{2} .
$$

In the first part of our simulations, we compare the pace of convergence of the reference sliding variable calculated according to (16) and (20). The results for different discretization periods are shown Figures 2 and 3. For reaching law (16), $q=3$, so the proportional factor $q$ T equals $0.3,0.15$, and 0.03 for $T=0.1 \mathrm{~s}, 0.05 \mathrm{~s}$, and $0.01 \mathrm{~s}$, respectively. On the other hand, in reaching law (20), the proportional factor $q=0.3$ is constant. It is worth mentioning that the initial condition $\mathbf{x}_{0}$ remained constant. However, due to different values of vector $\boldsymbol{c}$, we obtained different values of $s_{0}$.

As clearly visible from the presented graphs, the convergence pace with application of reaching law (16) decreases with decreasing discretization period. This means that the faster the system is sampled, the slower it converges to the sliding plane. On the other hand, reaching law (20) makes the reference converge to the sliding surface in approximately 10 steps. Consequently, reducing the discretization period $T$ results in shorter convergence time. For $T=0.1 \mathrm{~s}$, the reference sliding variable reaches the sliding plane in 1 second, for $T=0.05 \mathrm{~s}$ in $0.5 \mathrm{~s}$ and for $T=0.01 \mathrm{~s}$ in $0.1 \mathrm{~s}$. We have already noticed that the features of the reference sliding variable profile are transferred to the properties of the closed-loop system due to control (28). Consequently, it is obvious that the usage of reaching law (20) in the trajectory generator is more favourable, as it will ensure faster system's response.

In the second part of our simulations, we compare the reference sliding variable-based control for real disturbed system (62) with the original control strategy of Gao, with reaching law (13). As Gao's controller results in the quasisliding mode band width of $O(1)$ order, we only present the graphs obtained for $T=0.1 \mathrm{~s}$. We chose to generate the reference sliding variable with (20) as it provides faster convergence pace and apply reaching law (27) including the disturbance compensator. Therefore, for our strategy, the proportional term is $q=0.3$, and for the original strategy, $q T=0.3$. The resulting plots are presented in Figures 4-10. The trajectories resulting from the strategy of Gao are drawn 


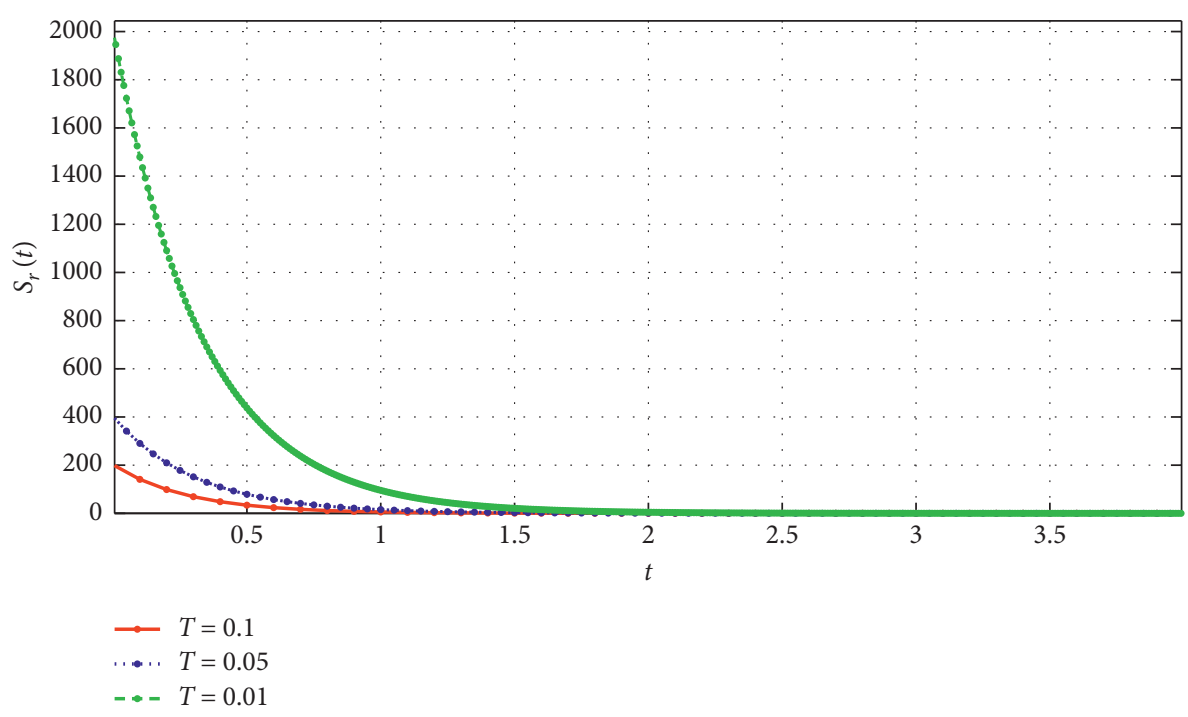

Figure 2: Profile of the reference sliding variable generated with reaching law (16).

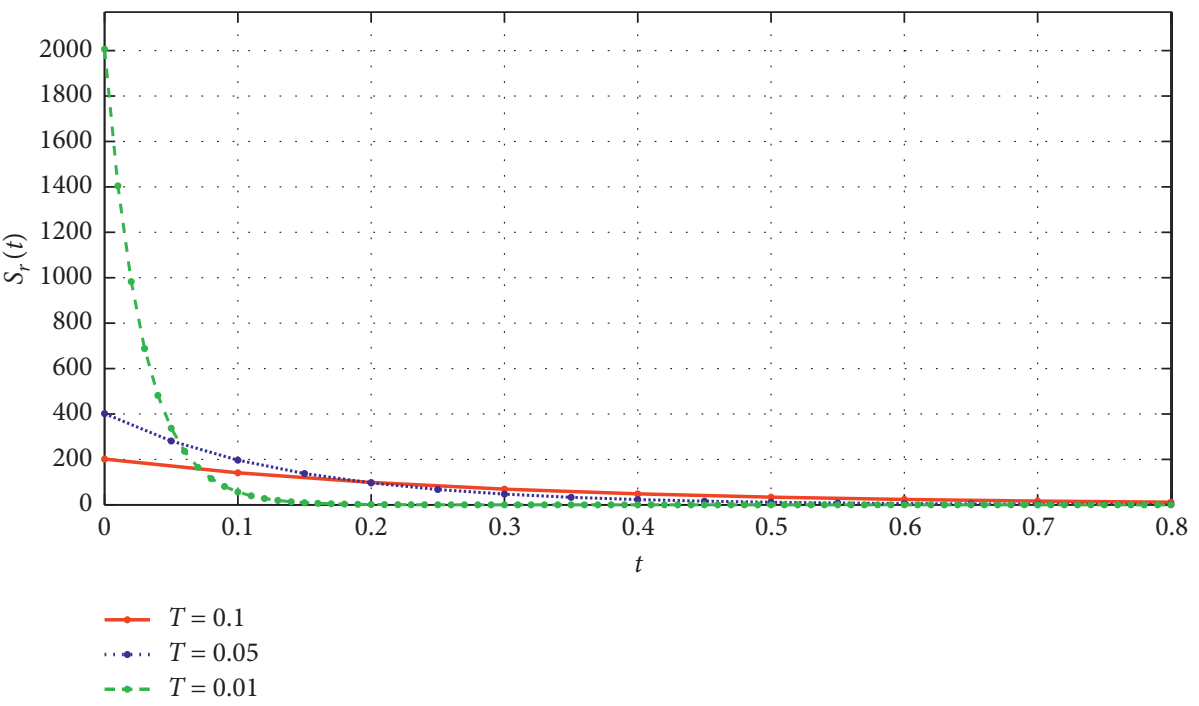

Figure 3: Profile of the reference sliding variable generated with reaching law (20).

with black dash-dot line, while the plots obtained with our control method are highlighted with red solid line. The comparison of the evolution of the sliding variables for the control of Gao and the proposed reference following control for $T=0.1 \mathrm{~s}$ is presented in Figures 4 and 5. Figure 5 is concentrated on the quasi-sliding mode band width.

It is clear that, while preserving the same discretization period $T=0.1 \mathrm{~s}$, the application of the reference sliding variable-based control and a disturbance compensator significantly reduced the width of the ultimate band. For Gao's control, in the quasi-steady state, the sliding variable is restricted by $|s(k)| \leq 0.65$, while in our control method, it holds that $|s(k)| \leq 0.061$. Next, in Figure 6, we compare the required control signals for both strategies. Lastly, Figures 7-10 depict the evolution of the state variables of the system. From the presented figures, it is clear that the state errors have been reduced as well. Therefore, our control algorithm ensures an improvement of the system's robustness without any enlargements of the control effort.

In the third part of our simulations, we focus on our new control method only. We present the impact of the chosen discretization period on the achieved quasi-sliding mode band width, so we consider all three discretization cases. The plots are presented with red solid line for $T=0.1 \mathrm{~s}$, blue dotted line for $T=0.05 \mathrm{~s}$, and green dashed line for $T=0.01 \mathrm{~s}$. Figure 11 presents how the convergence pace increases with the reduction of the discretization period. One may easily notice that the representative point of the system reaches the ultimate band in shortest time for $T=0.01 \mathrm{~s}$. Next, Figures 12-14 depict the changes of the obtained quasi- 


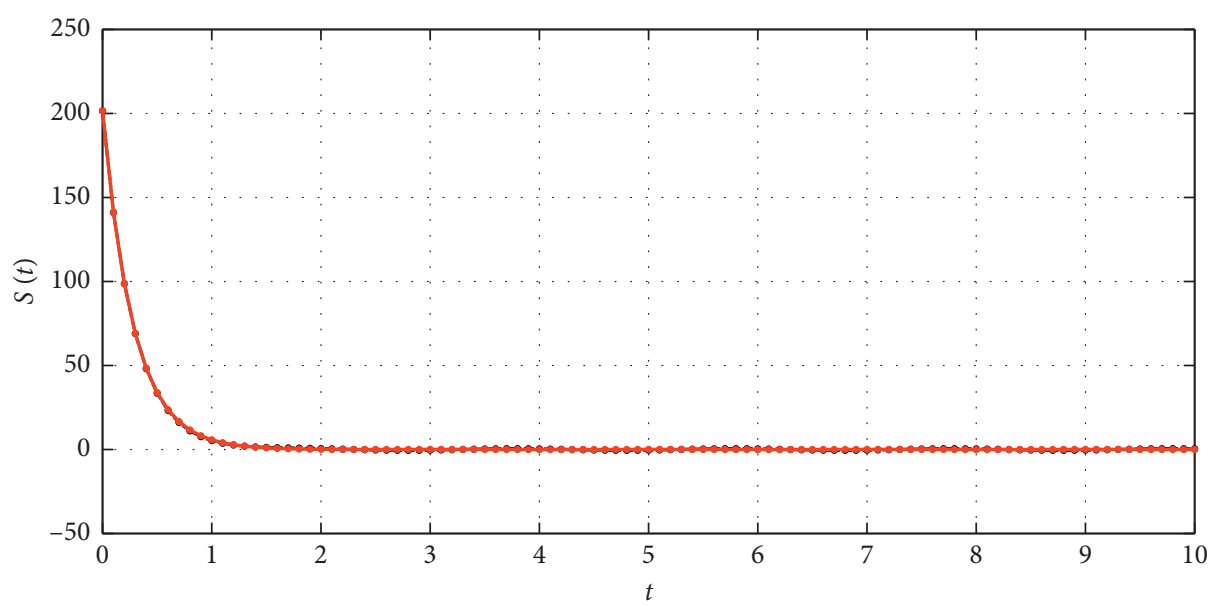

$\rightarrow$ - Sliding variable for the strategy of Gao

$\rightarrow$ Sliding variable with the disturbance compensator

Figure 4: Evolution of the sliding variable for $T=0.1 \mathrm{~s}$.

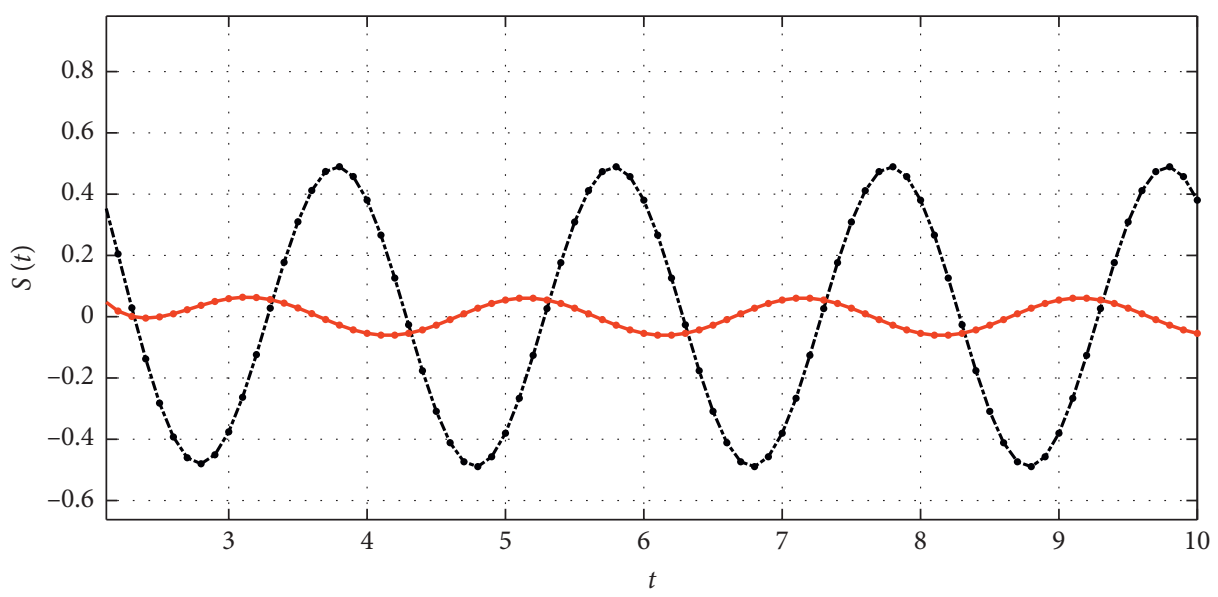

Sliding variable for the strategy of Gao

Sliding variable with the disturbance compensator

Figure 5: Evolution of the sliding variable for $T=0.1 \mathrm{~s}$ in the quasi-steady state.

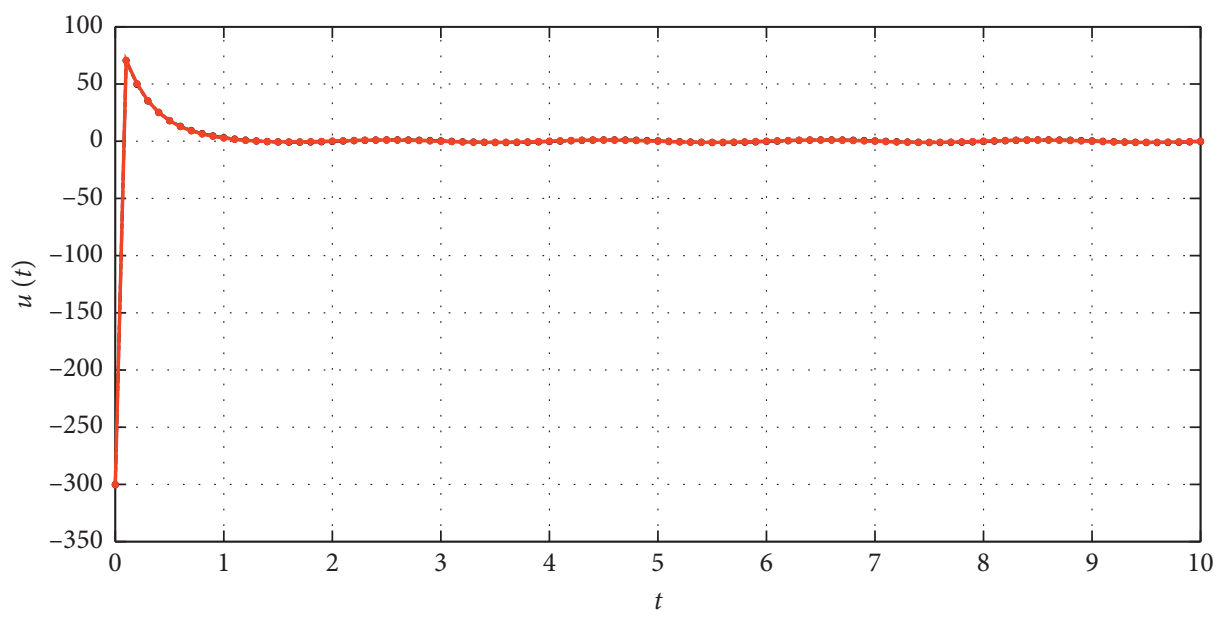

$\rightarrow$ - Control signal for the strategy of Gao

$\longrightarrow$ Control signal with the disturbance compensator

Figure 6: Control signal for $T=0.1 \mathrm{~s}$. 


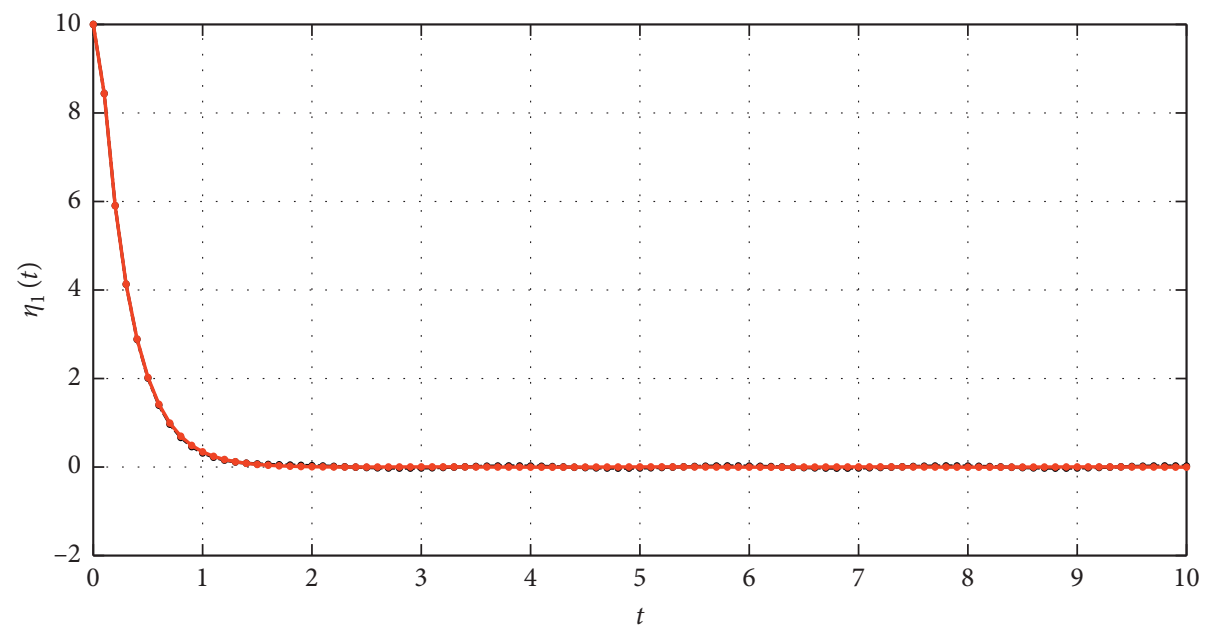

$\rightarrow-\eta_{1}$ for the strategy of Gao

$\rightarrow \eta_{1}$ with the disturbance compensator

FIgURE 7: Evolution of the first state variable for $T=0.1 \mathrm{~s}$.

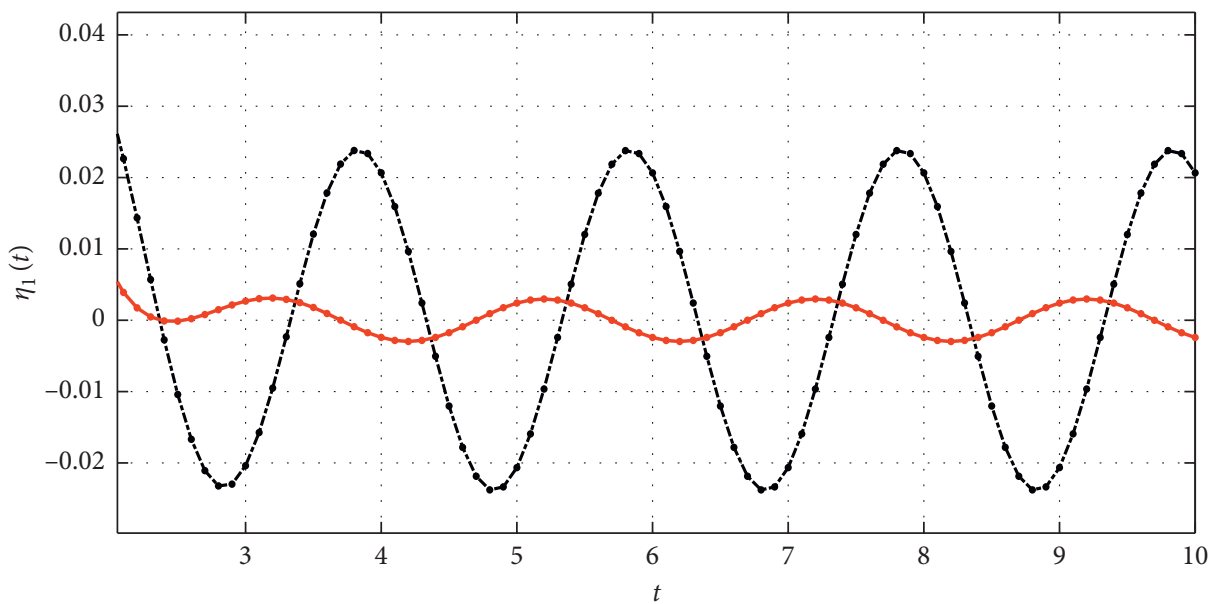

- - $\eta_{1}$ for the strategy of Gao

$\rightarrow \eta_{1}$ with the disturbance compensator

FIGURE 8: First state variable in the quasi-steady state for $T=0.1 \mathrm{~s}$.

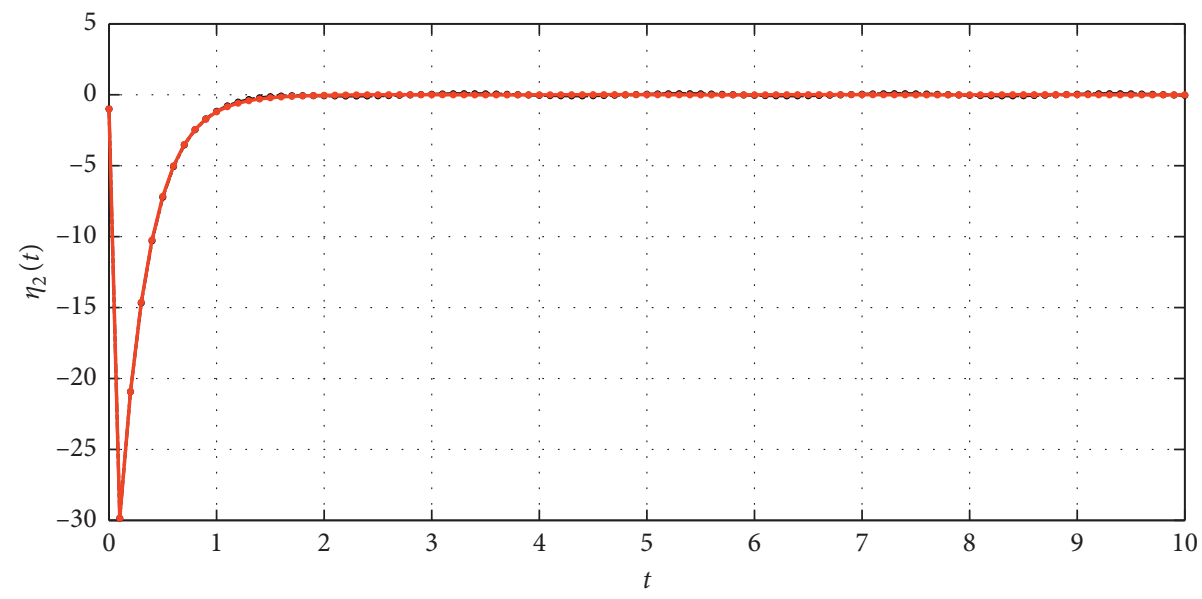

$\rightarrow-\eta_{2}$ for the strategy of Gao

$\rightarrow \eta_{2}$ with the disturbance compensator

FIgURE 9: Evolution of the second state variable for $T=0.1 \mathrm{~s}$. 


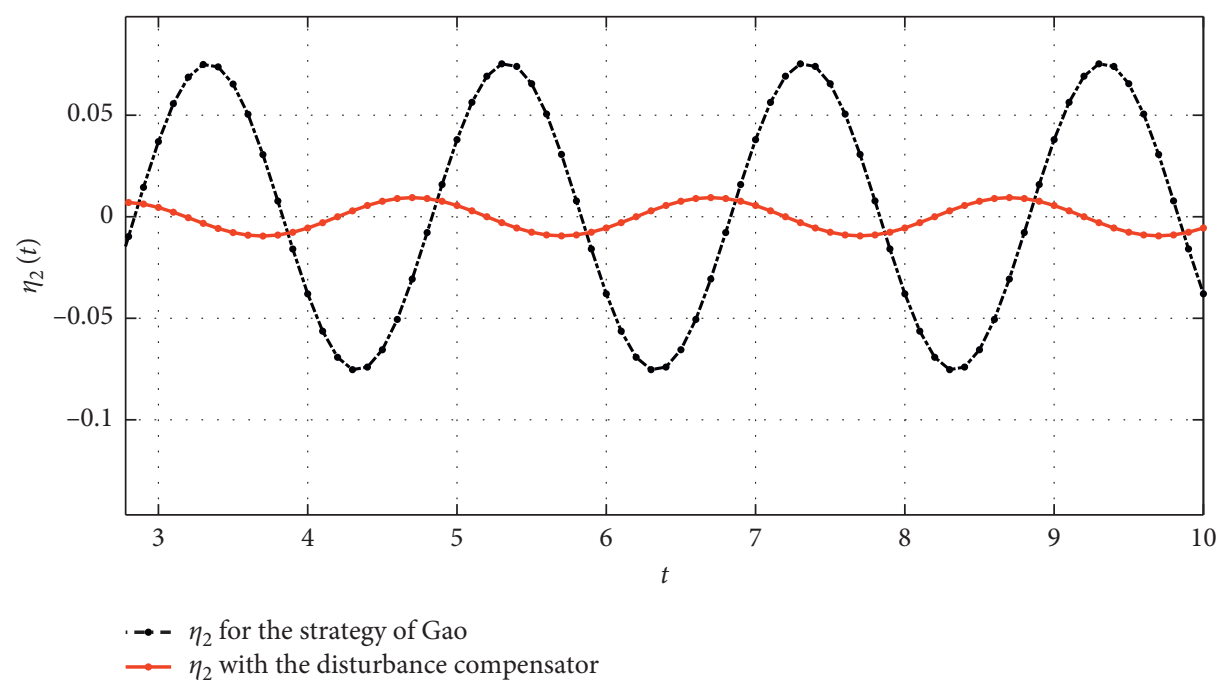

FIgURE 10: Second state variable in the quasi-steady state for $T=0.1 \mathrm{~s}$.

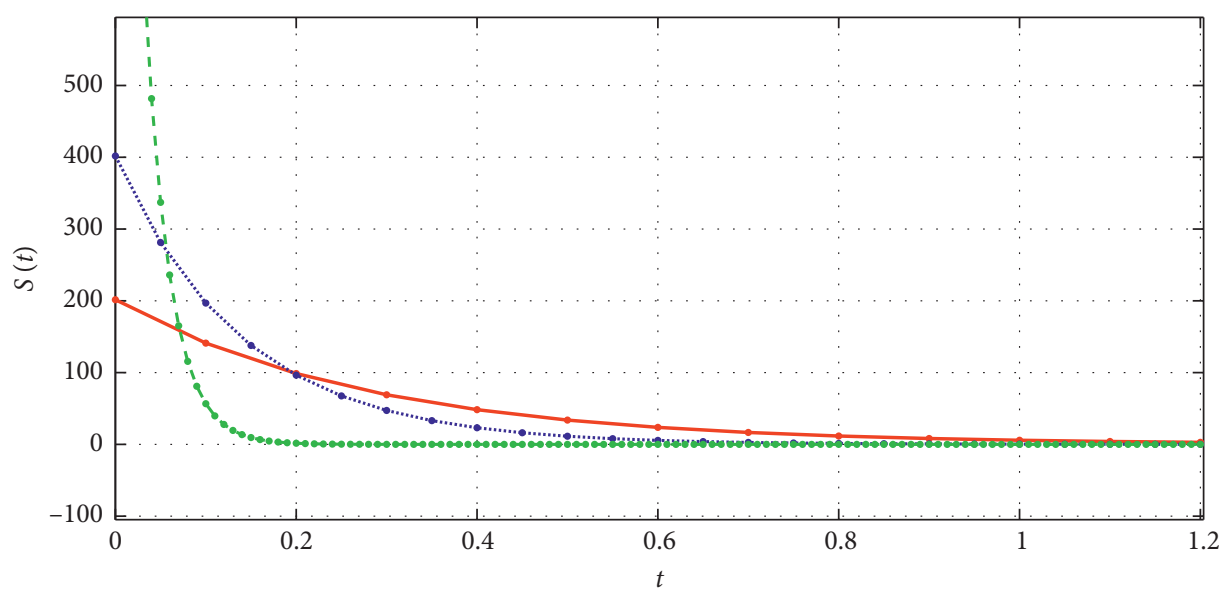

$$
\begin{aligned}
\because T & =0.1 \\
\cdots T & =0.05 \\
\because T & =0.01
\end{aligned}
$$

FIGURE 11: Comparison of the convergence pace with different discretization periods.

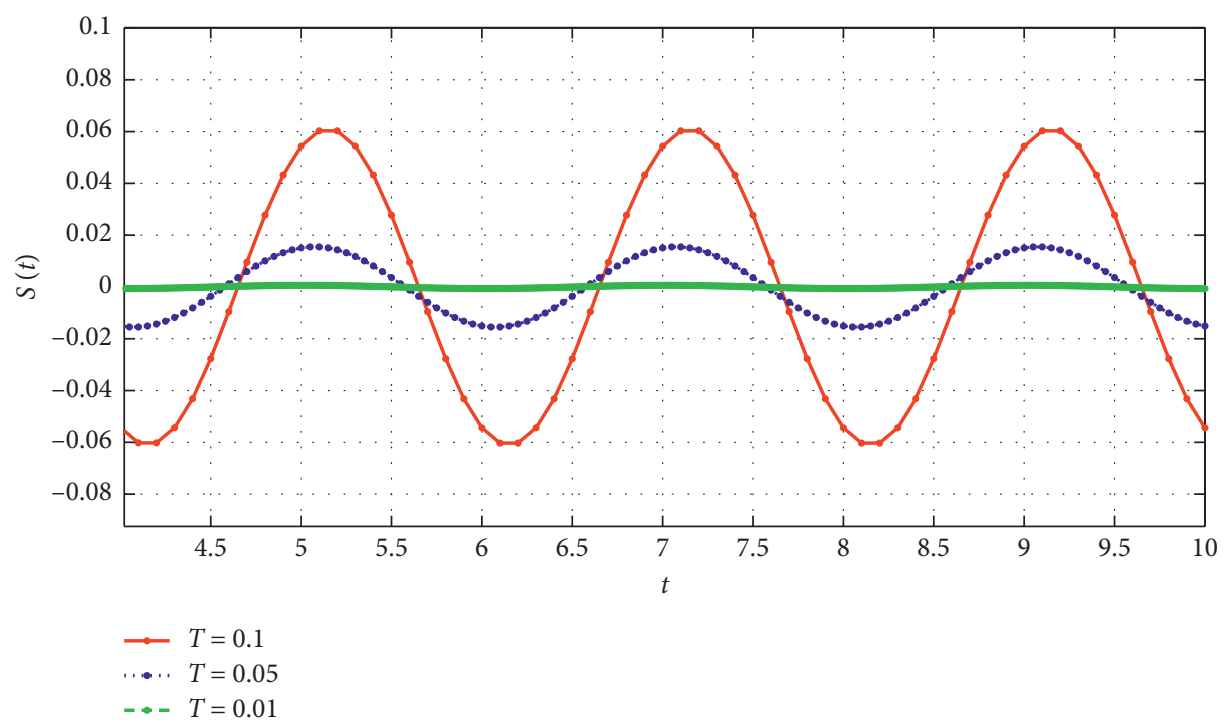

FIGURE 12: Sliding variable in the quasi-steady state for the proposed strategy for different discretization periods. 


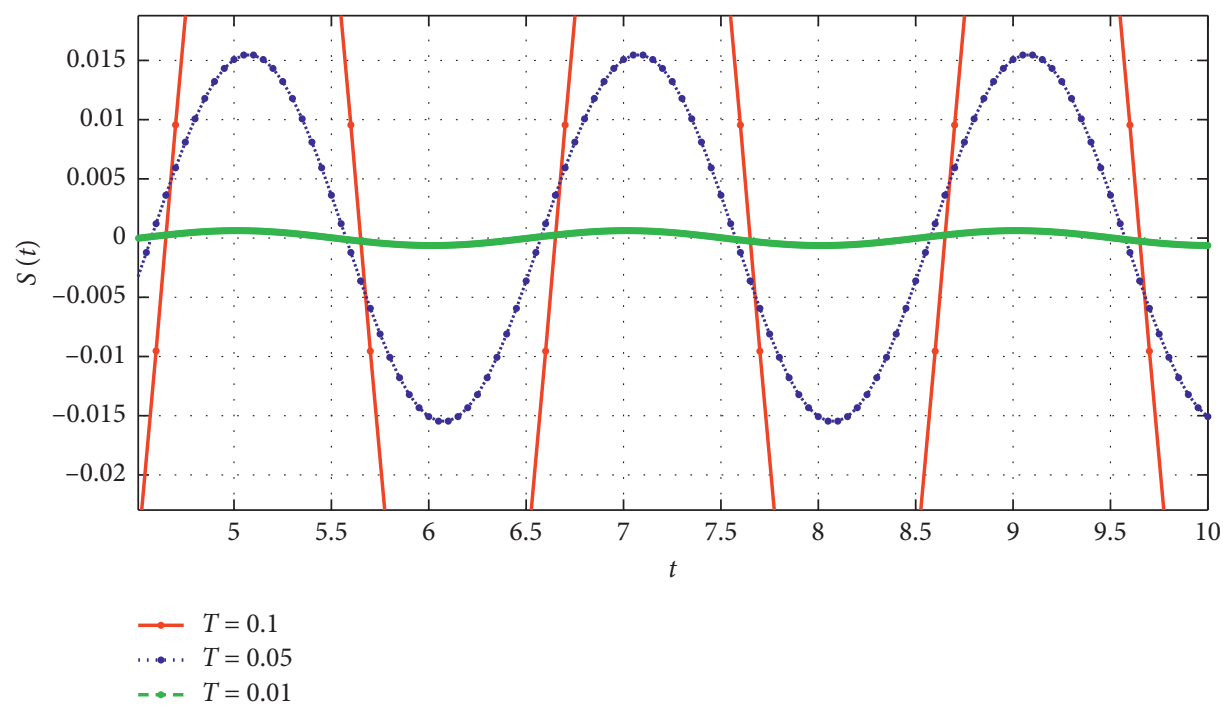

FIGURE 13: Sliding variable in the quasi-steady state for the proposed strategy for different discretization periods (zoom for $T=0.05 \mathrm{~s}$ ).

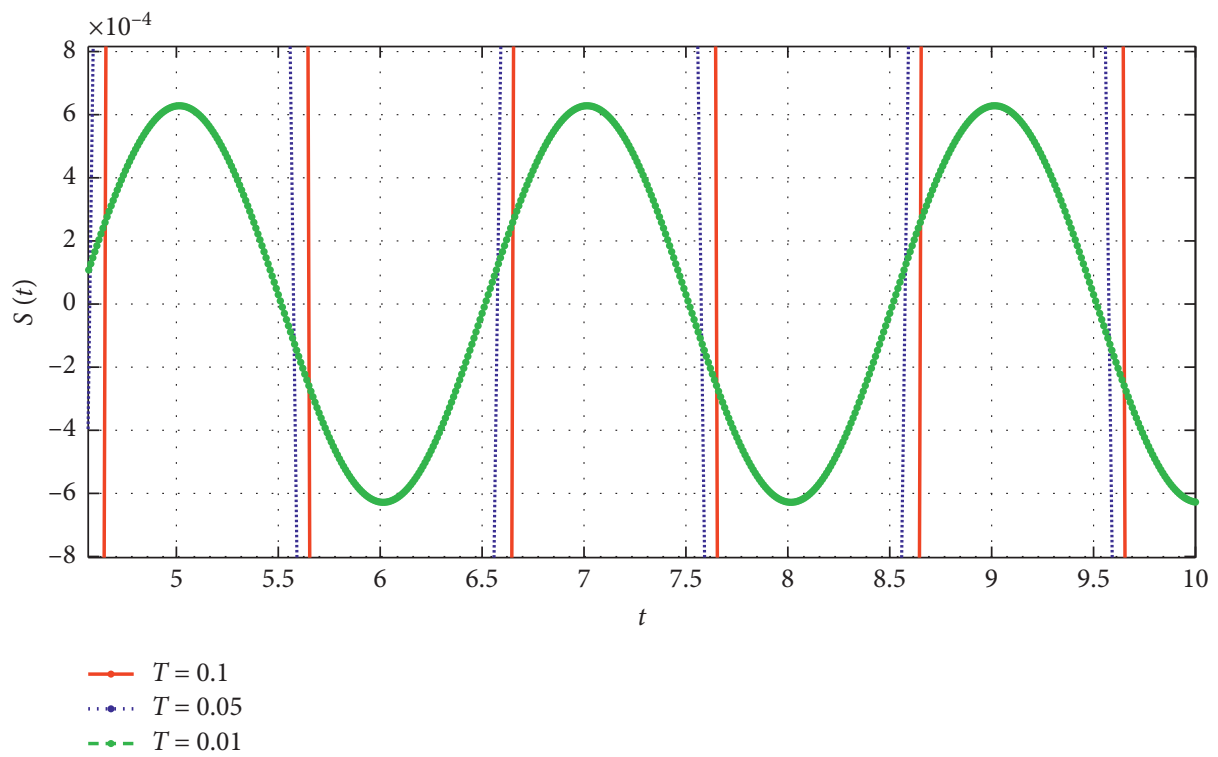

FIGURE 14: Sliding variable in the quasi-steady state for the proposed strategy for different discretization periods (zoom for $T=0.01 \mathrm{~s}$ ).

sliding mode band width. The exact values compared to the original control of Gao can be found in Table 1.

From the presented figures, one may easily verify that the width of the ultimate band achieved with the proposed control method is of $O\left(T^{2}\right)$ order, while in Gao's strategy, it remains of $O(1)$ order. Figure 11 also proves that our new control strategy ensures faster convergence with the reduction of the discretization period.
Lastly, we calculated the maximum state variables' error values for both strategies with different discretization periods. The exact values are presented in Figures 15 and 16 and in Table 1.

It is worth noticing that the calculated error values are the maximum values. The actual state variables' errors may be lower, as the disturbance is not maximal for the whole control process. 
TABLE 1: Comparison of the obtained quasi-sliding mode band widths and maximum state errors.

\begin{tabular}{lcc}
\hline Discretization period & Proposed new strategy & Original strategy \\
\hline Ultimate band width & & \\
$T=0.1 \mathrm{~s}$ & $2 \cdot 6.13 \cdot 10^{-2}=2 \cdot 6.13 \cdot T^{2}$ & $2 \cdot 0.65$ \\
$T=0.05 \mathrm{~s}$ & $2 \cdot 1.55 \cdot 10^{-2}=2 \cdot 6.2 \cdot T^{2}$ & \\
$T=0.01 \mathrm{~s}$ & $2 \cdot 6.2 \cdot 10^{-4}=2 \cdot 6.2 \cdot T^{2}$ \\
\hline Maximum error of $\eta_{1}$ & & \\
$T=0.1 \mathrm{~s}$ & $0.003=0.3 \cdot T^{2}$ \\
$T=0.05 \mathrm{~s}$ & $0.0004=0.16 \cdot T^{2}$ \\
$T=0.01 \mathrm{~s}$ & $0.000031=0.31 \cdot T^{2}$ \\
\hline Maximum error of $\eta_{2}$ & & \\
$T=0.1 \mathrm{~s}$ & $0.0605=6.05 \cdot T^{2}$ \\
$T=0.05 \mathrm{~s}$ & $0.01538=6.15 \cdot T^{2}$ \\
$T=0.01 \mathrm{~s}$ & $0.00062=6.2 \cdot T^{2}$ \\
\hline
\end{tabular}

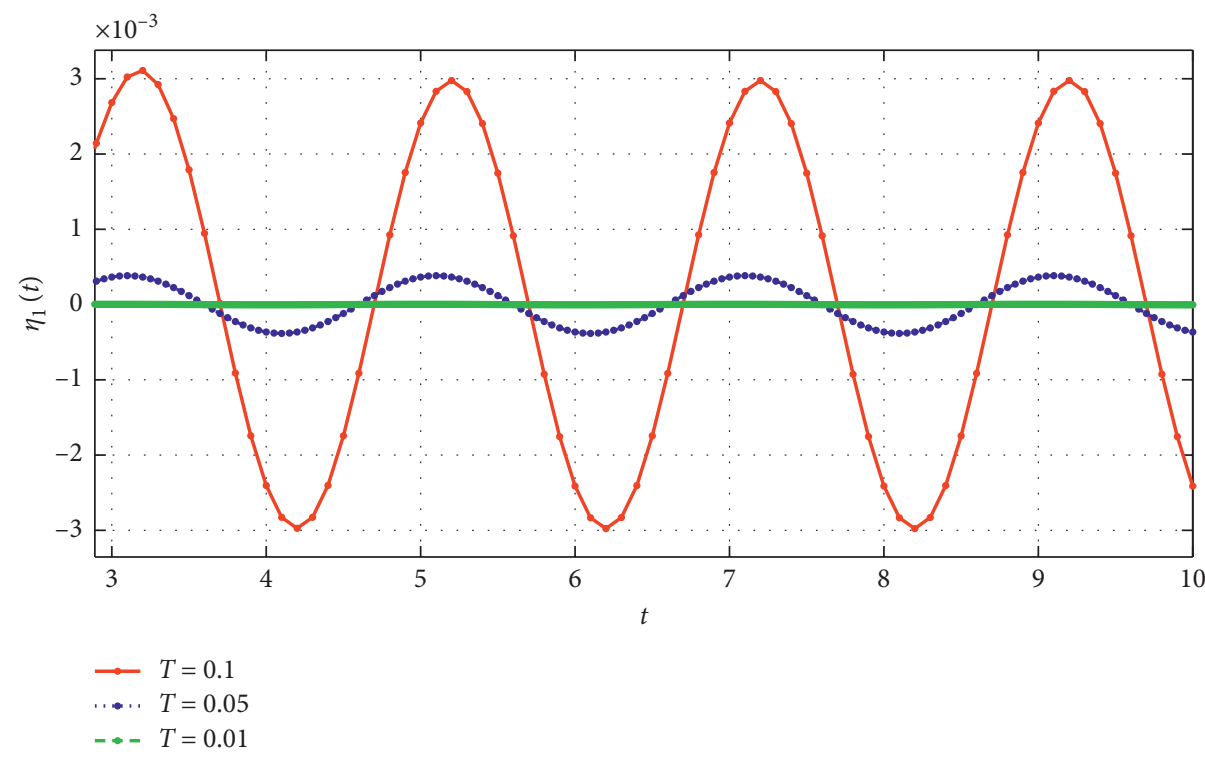

Figure 15: Evolution of the first state variable for the proposed strategy for different discretization periods.

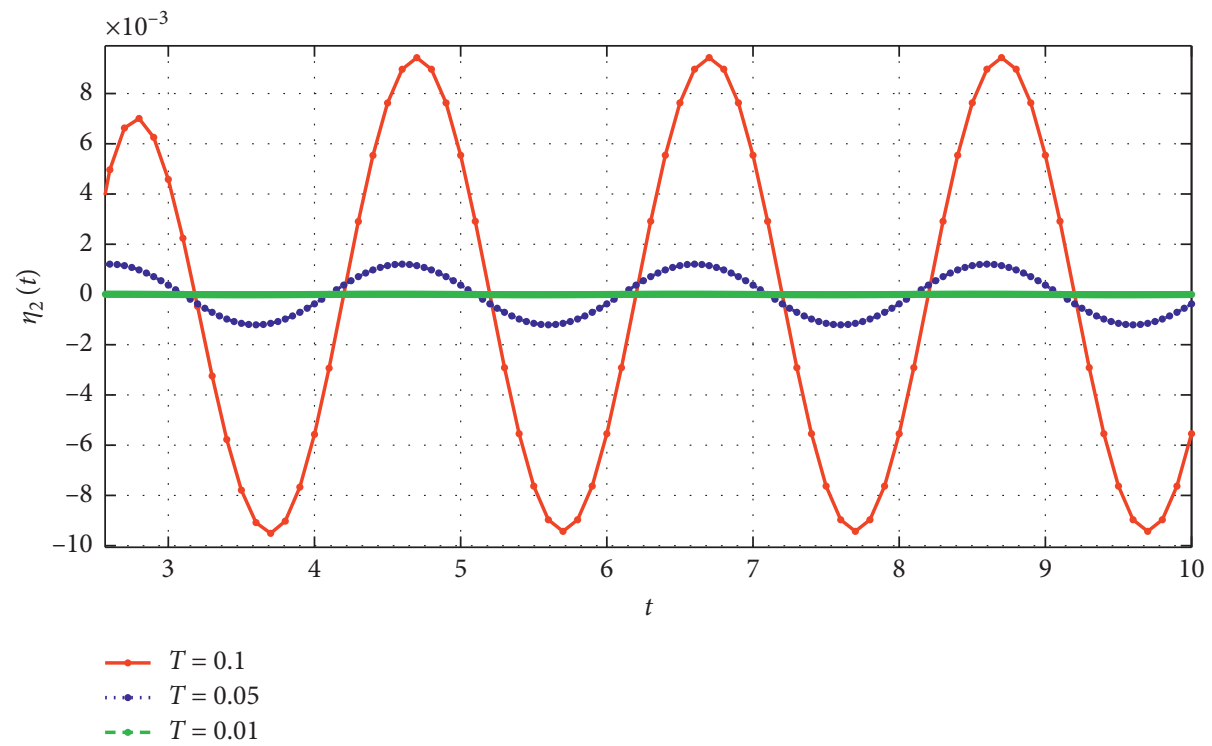

Figure 16: Evolution of the second state variable for the proposed strategy for different discretization periods. 


\section{Conclusions}

This study presented a new approach to sliding mode control of sampled data systems. We adopted the nonswitching type definition of the quasi-sliding mode and proposed a new reference sliding variable-based controller. The key of our control method is to generate the desired evolution of the sliding variable externally and further utilize it to control the plant. We proved that this approach limits the influence of external disturbance on the system to one control step and therefore improves the system's robustness. Following the seminal work of Gao et al., we considered two different reaching laws for generation of the demand sliding variable profile and analysed their influence on the system's pace of convergence. Next, we incorporated the reference profile into the reaching law for the real disturbed system. We proved that our control results in the ultimate band width of $O(T)$ order, so it decreases with the reduction of the discretization period. Moreover, thanks to elimination of the switching term from the reaching law, the problem of chattering is minimized. Next, we supplemented our control law with a disturbance compensation algorithm, which enabled a reduction of the quasi-sliding mode band width to $O\left(T^{2}\right)$ order. Lastly, we also proved that in the sliding phase, all the state variables' errors become restricted to the band of $O\left(T^{2}\right)$ as well. To conclude the study, we carried out a thorough simulation example, which demonstrated the benefits of the presented control method.

\section{Data Availability}

The data used to support the findings of this study are included within the article.

\section{Conflicts of Interest}

The author declares that there are no conflicts of interest regarding the publication of this paper.

\section{Acknowledgments}

This study was supported by Institute of Automatic Control of Łódź University of Technology.

\section{References}

[1] U. Itkis, Control Systems of Variable Structures, Wiley, New York, NY, USA, 1976.

[2] V. Utkin, "Variable structure systems with sliding modes," IEEE Transactions on Automatic Control, vol. 22, no. 2, pp. 212-222, 1977.

[3] V. I. Utkin, "Variable structure systems: present and future," Automation and Remote Control, vol. 44, no. 9, pp. 1105-1220, 1984.

[4] J. Y. Hung, W. Gao, and J. C. Hung, "Variable structure control: a survey," IEEE Transactions on Industrial Electronics, vol. 40, no. 1, pp. 2-22, 1993.

[5] A. Levant, "Sliding order and sliding accuracy in sliding mode control," International Journal of Control, vol. 58, no. 6, pp. 1247-1263, 1993.
[6] C. Edwards and S. Spurgeon, Sliding Mode Control: Theory and Applications, Taylor \& Francis, London, UK, 1998.

[7] A. Bartoszewicz and A. Nowacka-Leverton, "SMC without the reaching phase - the switching plane design for the thirdorder system," IET Control Theory \& Applications, vol. 1, no. 5, pp. 1461-1470, 2007.

[8] B. Draženović, "The invariance conditions in variable structure systems," Automatica, vol. 5, no. 3, pp. 287-295, 1969.

[9] O. Kaynak and A. Denker, "Discrete-time sliding mode control in the presence of system uncertainty," International Journal of Control, vol. 57, no. 5, pp. 1177-1189, 1993.

[10] W.-C. Su, S. V. Drakunov, and U. Ozguner, "An $O\left(T^{2}\right)$ boundary layer in sliding mode for sampled-data systems," IEEE Transactions on Automatic Control, vol. 45, no. 3, pp. $482-485,2000$.

[11] C. Milosavljević, "General conditions for the existence of a quasi-sliding mode on the switching hyperplane in discrete variable structure systems," Automation and Remote Control, vol. 46, no. 3, pp. 307-314, 1985.

[12] S. Sarpturk, Y. Istefanopulos, and O. Kaynak, "On the stability of discrete-time sliding mode control systems," IEEE Transactions on Automatic Control, vol. 32, no. 10, pp. 930-932, 1987.

[13] U. Kotta, S. Z. Sarpturk, and Y. Istefanopulos, "Comments on "On the stability of discrete-time sliding mode control systems" [with reply]," IEEE Transactions on Automatic Control, vol. 34, no. 9, pp. 1021-1022, 1989.

[14] H. Sira-Ramirez, "Non-linear discrete variable structure systems in quasi-sliding mode," International Journal of Control, vol. 54, no. 5, pp. 1171-1187, 1991.

[15] K. Furuta, "Sliding mode control of a discrete system," Systems \& Control Letters, vol. 14, no. 2, pp. 145-152, 1990.

[16] K. Furuta, "VSS type self-tuning control," IEEE Transactions on Industrial Electronics, vol. 40, no. 1, pp. 37-44, 1993.

[17] S. V. Drakunov and V. I. Utkin, "On discrete-time sliding modes," IFAC Proceedings Volumes, vol. 22, no. 3, pp. 273-278, 1989.

[18] G. Bartolini, A. Ferrara, and V. I. Utkin, "Adaptive sliding mode control in discrete-time systems," Automatica, vol. 31, no. 5, pp. 769-773, 1995.

[19] W. B. Gao, Y. Wang, and A. Homaifa, "Discrete-time variable structure control systems," IEEE Transactions on Industrial Electronics, vol. 42, no. 2, pp. 117-122, 1995.

[20] A. Bartoszewicz, "Remarks on 'Discrete-time variable structure control systems'," IEEE Transactions on Industrial Electronics, vol. 43, pp. 235-238, 1996.

[21] S. Mija and T. Susy, "Reaching law based sliding mode control for discrete MIMO systems," IEEE International Conference on Control, Automation, Robotics \& Vision, pp. 1291-1296, 2010.

[22] B. Veselic, B. Perunicic-Drazenovic, and Č. Milosavljevic, "Improved discrete-time sliding-mode position control using Euler velocity estimation," IEEE Transactions on Industrial Electronics, vol. 57, no. 11, pp. 3840-3847, 2010.

[23] G. Golo and Č. Milosavljević, "Robust discrete-time chattering free sliding mode control," Systems \& Control Letters, vol. 41, no. 1, pp. 19-28, 2000.

[24] Č. Milosavljević, B. Peruničić-Draženović, B. Veselić, and D. Mitić, "A new design of servomechanisms with digital sliding mode," Electrical Engineering, vol. 89, no. 3, pp. 233-244, 2007.

[25] A. Bartoszewicz, "Discrete-time quasi-sliding-mode control strategies," IEEE Transactions on Industrial Electronics, vol. 45, no. 4, pp. 633-637, 1998. 
[26] S. Chakrabarty and B. Bandyopadhyay, "Minimum ultimate band design of discrete sliding mode control," Asian Journal of Control, vol. 17, no. 5, pp. 1189-1897, 2015.

[27] A. Bartoszewicz and P. Lesniewski, "New switching and nonswitching type reaching laws for SMC of discrete time systems," IEEE Transactions on Control Systems Technology, vol. 24, no. 2, pp. 670-677, 2016.

[28] K. Abidi, J.-X. Xu, and Y. Xinghuo, "On the discrete-time integral sliding-mode control," IEEE Transactions on Automatic Control, vol. 52, no. 4, pp. 709-715, 2007.

[29] H. Ma, J. Wu, and Z. Xiong, "A novel exponential reaching law of discrete-time sliding-mode control," IEEE Transactions on Industrial Electronics, vol. 64, no. 5, pp. 3840-3850, 2017.

[30] J. Zhang, N. Zhang, G. Shen, and Y. Xia, "Analysis and design of chattering-free discrete-time sliding mode control," International Journal of Robust and Nonlinear Control, vol. 29, no. 2, pp. 6572-6581, 2019.

[31] B. Bandyopadhyay and S. Janardhanan, Discrete-Time Sliding Mode Control: A Multirate Output Feedback Approach, Springer-Verlag, Berlin, Germany, 2006.

[32] S. Janardhanan and V. Kariwala, "Multirate-output-feedbackbased LQ-optimal discrete-time sliding mode control," IEEE Transactions on Automatic Control, vol. 53, no. 1, pp. 367-373, 2008.

[33] B. Bandyopadhyay and A. K. Behera, "Event-triggered sliding mode control," Studies in Systems, Decision and Control, vol. 139, Springer, Berlin, Germany, 2018.

[34] S. Chakrabarty, B. Bandyopadhyay, J. Moreno, and L. Fridman, "Discrete sliding mode control for systems with arbitrary relative degree output," in Proceedings of the 14th International Workshop on Variable Structure Systems (VSS), pp. 160-165, Nanjing, China, 2016.

[35] S. Chakrabarty and A. Bartoszewicz, "Improved robustness and performance of discrete time sliding mode control systems," ISA Transactions, vol. 65, no. 1, pp. 143-149, 2016.

[36] A. Bartoszewicz and P. Latosiński, "Reaching law for DSMC systems with relative degree 2 switching variable," International Journal of Control, vol. 90, no. 8, pp. 1626-1638, 2017.

[37] A. Bartoszewicz and P. Latosinski, "Generalization of Gao's reaching law for higher relative degree sliding variables," IEEE Transactions on Automatic Control, vol. 63, no. 9, pp. 31733179, 2018. 